\title{
Retinal vascular changes in hypertensive patients in Ibadan, Sub-Saharan Africa
}

This article was published in the following Dove Press journal:

International Journal of General Medicine

4 August 2016

Number of times this article has been viewed

\author{
Sunday Tunji Oluleye' \\ Bolutife Ayokunu Olusanya' \\ Abiodun Moshood Adeoye ${ }^{2}$ \\ 'Department of Ophthalmology, \\ ${ }^{2}$ Department of Medicine, College of \\ Medicine, University of Ibadan and \\ University College Hospital, Ibadan, \\ Nigeria
}

Background: Earlier studies in Nigeria reported the rarity of retinal vascular changes in hypertensives. The aim of this study was to describe the various retinal vascular changes in the hypertensive patients of Nigeria.

Patients and methods: Nine hundred and three hypertensive patients were studied. This study was approved by the ethical and research committee of the University of Ibadan and University College Hospital, Ibadan, Nigeria. Blood pressure and anthropometric measurements were measured. Cardiac echocardiography was performed on 156 patients. All patients had dilated fundoscopy and fundus photography using the Kowa portable fundus camera and an Apple iPhone with 20 D lens. Statistical analysis was done with Statistical Packages for the Social Sciences (Version 21).

Results: The mean age of patients was 57 years with a male:female ratio of 1 . No retinopathy was found in $556(61.5 \%)$ patients. In all, 175 (19.4\%) patients had features of hypertensive retinopathy. Retinal vascular occlusion was a significant finding in 121 patients $(13.4 \%)$, of which branch retinal vein occlusion, 43 (4.7\%), and central retinal vein occlusion, 30 (3.3\%), were the most prominent ones in cases. Hemicentral retinal vein occlusion, 26 (2.9\%), and central retinal artery occlusion, 17 (1.9\%), were significant presentations. Other findings included nonarteritic anterior ischemic optic neuropathy in five $(0.6 \%)$ patients, hypertensive choroidopathy in seven $(0.8 \%)$ patients, and hemorrhagic choroidal detachment in five $(0.6 \%)$ patients. Left ventricular (LV) geometry was abnormal in 85 (55.5\%) patients. Concentric remodeling, eccentric hypertrophy, and concentric hypertrophy were observed in 43 (27.6\%), 26 (17.2\%), and $15(9.7 \%)$ patients, respectively. LV hypertrophy was found in $42(27 \%)$ patients, while 60 (39\%) patients had increased relative wall thickness. In this study, bivariate analysis showed a correlation between LV relative wall thickness and severity of retinopathy in both eyes (Spearman's coefficient $0.6 ; P=0.0004$ ).

Conclusion: Hypertensive retinal vascular changes are not rare in Ibadan.

Keywords: hypertensive retinopathy, retinal vascular occlusion, retinal vascular changes, left ventricular wall thickness, Ibadan

\section{Introduction}

Systemic hypertension is very common in Nigeria. ${ }^{1}$ Complications of systemic hypertension in Nigeria include ischemic heart disease, stroke, congestive heart disease, and renal failure. $^{2-7}$ The retinal vascular abnormalities from systemic hypertension are usually a pointer to systemic morbidity and mortality. ${ }^{8}$ There is a strong association between hypertensive retinopathy and blood pressure. ${ }^{9}$ Systemic hypertension produces vascular wall dam-
Department of Ophthalmology, College of Medicine, University of lbadan and University College Hospital, PMB 5116 , Ibadan, Nigeria

Tel +2348023265594

Email t_oluleye@yahoo.co.uk 
age in various organs such as the heart, eye, brain, and kidney. Systemic hypertension also produces damage in the optic nerve, choroid, and retina known as neuropathy, choroidopathy, and retinopathy, respectively. Hypertensive retinal changes include vascular narrowing, arteriovenous changes, occlusion, and exudation. In severe hypertension, increased retinal hemorrhages and optic nerve head swelling may be observed. ${ }^{10}$

A previous study showed a close relationship between retina vascular changes and ventricular hypertrophy. ${ }^{11}$ Proteinuria is also strongly associated with advanced retinopathy. ${ }^{12}$ Microaneurysms, cotton wool spots, and retinal hemorrhages can predict stroke, cardiac morbidity, and mortality, ${ }^{13}$ whereas mild retinopathic changes are not strongly associated with target organ damage. ${ }^{14,15}$ However, retinal changes of any grade were reported to have a moderate accuracy in predicting coronary heart disease and, hence, may be used as an early screening tool in a resource-poor setting. ${ }^{16}$ The high rate of hypertensive retinopathy in Afro-Caribbeans may be due to other factors such as age rather than blood pressure alone. ${ }^{17}$ Controversy exists on the prevalence of hypertensive retinopathy in Nigeria. ${ }^{18,19}$ There is paucity of data; hence, there is the need to revisit the topic. The aim of this study was to describe the retinal vascular abnormalities in patients aged $\geq 40$ years who attended the hypertension clinic of the medical outpatient department of the University College Hospital, Ibadan.

\section{Patients and methods Study design}

This was a cross-sectional hospital-based study. The descriptive component of this study included the prevalence and the description of the types of retinopathic changes, while the analytical component included the relationship between severity of retinopathy and the predictors of cardiovascular complications.

\section{Study population}

A total of 903 consecutive hypertensive patients satisfying the study criteria were studied. The sample size was calculated based on the criteria laid down for health studies. ${ }^{20}$

\section{Data collection and examination protocol}

This study was done in accordance with the Declaration of Helsinki and the protocol was approved by the ethical and research committee of the University of Ibadan and University College Hospital, Ibadan, Nigeria. Written informed consent was obtained from all patients. Blood pressure and anthropometric measurements (height, weight, body mass index, hip-waist ratio) were measured using standardized protocols by the physician investigator.

Echocardiography was performed on 156 patients in view of the cost. Standardized measurements were taken as stipulated by the American Heart Association, European Association of Echocardiography, and other relevant cardiac committees. ${ }^{21}$ Left ventricular hypertrophy (LVH) was considered present if the left ventricular mass index (LVMI) was $\geq 51 \mathrm{~g} / \mathrm{m}^{2}$.

$$
\begin{array}{r}
\text { Left ventricular mass }=0.8 \times(1.04 \times[\text { LVEDD }+ \text { PWTd }+ \\
\text { SWTd }] 3-[\text { LVEDD }] 3)+0.6
\end{array}
$$

where LVEDD is the left ventricular (LV) end-diastolic diameter, PWTd is the diastolic posterior wall thickness, and SWTd is the diastolic septal wall thickness. LV mass is commonly indexed by the body surface area to define LVMI $\left(\mathrm{g} / \mathrm{m}^{2}\right)$.

Relative wall thickness (RWT) was calculated as:

$$
\mathrm{RWT}=2 \frac{\mathrm{PWTd}}{\mathrm{LVEDD}}
$$

where PWTd is the diastolic posterior wall thickness and LVEDD is the LV end-diastolic diameter. Increased wall thickness was present when RWT $>0.45$.

LV geometry was stratified using LVMI and RWT: normal geometry was normal LVMI and RWT, concentric remodeling was normal LVMI and increased RWT, eccentric hypertrophy was increased LVMI and RWT $<0.45$, and concentric hypertrophy was increased LVMI and RWT $\geq 0.45$.

Dilated fundoscopy with indirect ophthalmoscope and noncontact slit lamp biomicroscopy was done with topical $0.5 \%$ tropicamide on all patients by the ophthalmic investigator to determine the presence of retinal vascular lesions, such as retinal arteriolar narrowing, hemorrhages, cotton wool spots, exudates, arteriovenous nicking, vascular occlusions, and silver wiring and copper wiring of vessels. Fundus photographs of retinal vascular changes were taken with the Kowa portable fundus camera (Kowa Optimed Deutschland GmbH, Düsseldorf, Germany) and an iPhone 5 (Apple Inc., Cupertino, CA, USA) with 20 D lens adapted to capture retinal images. The data were recorded in the data sheet prepared for this study. Analysis was done using percentages and proportions. Statistical Packages for the Social Sciences (Version 21; IBM Corporation, Armonk, NY, USA) was used to test the strength of associations. 
Table I Retinal vascular changes in hypertensive patients in Ibadan

\begin{tabular}{lll}
\hline Retinal vascular changes & Frequency & Percentage \\
\hline Hypertensive retinopathy & 175 & 19.4 \\
Grade I & 100 & \\
Grade 2 & 63 & \\
Grade 3 & 10 & \\
Grade 4 & 2 & \\
Retinal vascular occlusion & 121 & 13.4 \\
CRVO & 30 & \\
Branch retinal vein occlusion & 43 & \\
Hemicentral vein occlusion & 26 & \\
Central retinal artery occlusion & 17 & \\
Branch retinal artery occlusion & 3 & \\
Combined retinal artery and vein & 2 & \\
occlusion & & \\
Optic atrophy (HT optic neuropathy) & 23 & 2.5 \\
RAM & 11 & 1.2 \\
Anterior ischemic optic neuropathy & 5 & 0.6 \\
Hypertensive choroidopathy & 7 & 0.8 \\
Hemorrhagic choroidal detachment & 5 & 0.6 \\
No retinopathy & 556 & 61.5 \\
Total & 903 & 100 \\
\hline Abrevation CRVO, cen &
\end{tabular}

Abbreviations: CRVO, central retinal vein occlusion; $\mathrm{HT}$, hypertensive; RAM, retinal artery macroaneurysm.

\section{Results}

Nine hundred and three patients were studied. The mean age of the patients was 57 years with a male:female ratio of 1 .

No retinopathy was found in 556 patients $(61.5 \%)$. Of those with retinal vascular abnormalities, 175 (19.4\%) had features of hypertensive retinopathy. Retinal vascular occlusion was a significant finding in 121 patients $(13.4 \%)$, of which branch retinal vein occlusion, 43 (4.7\%), and central retinal vein occlusion (CRVO), 30 (3.3\%), were the most prominent ones in cases (Table 1). Hemicentral retinal vein occlusion, 26 (2.9\%), and central retinal artery occlusion, 17 (1.9\%), were significant presentations. Two cases of combined retinal artery occlusion and vein occlusion occurred in uncontrolled hypertensive patients.

Hypertensive (HT) optic neuropathy with optic atrophy was found in $23(2.5 \%)$ patients, while retinal artery macroaneurysm (RAM) was detected in eleven $(1.2 \%)$ patients with poor blood pressure control (Figure 1). Other findings included nonarteritic anterior ischemic optic neuropathy, five $(0.6 \%)$ patients; hypertensive choroidopathy, seven $(0.8 \%)$ patients; and hemorrhagic choroidal detachment, five $(0.6 \%)$ patients.

In the 156 patients who had echocardiography, we tried to find the relationship between ventricular geometry, wall thickness, and the severity of retinopathy. The LV geometry was abnormal in $85(54.5 \%)$ subjects with the frequency of concentric remodeling, eccentric hypertrophy, and concentric hypertrophy in the subjects at 43 (27.6\%), 26 (17.2\%), and 15 (9.7\%), respectively. Echographic LVH was found in $42(27 \%)$ subjects, while 60 (39\%) had increased RWT. There was a positive correlation between LV RWT and severity of retinopathy in both eyes in this study. Negative correlation existed between LVMI and retinopathy. There was no correlation between LV geometric pattern and retinopathy.

\section{Discussion}

This study revealed a significant level of hypertensive retinopathy and retinal vascular changes. Approximately $40 \%$ of the screened patients presented with retinal vascular changes due to systemic hypertension. There appeared to be a changing trend as a previous study from Ibadan found hypertensive retinopathy to be rare.$^{18}$ Ladipo $^{19}$ in Lagos found hypertensive retinopathy in $>70 \%$ of 350 patients screened. Busari et $\mathrm{al}^{22}$ in a recent report also found significant proportion of hypertensive retinopathy in Ilorin. Another report from a neighboring state found a prevalence of $11 \%{ }^{23}$ and confirmed significant proportion of the condition in Nigerians. It is not surprising to find a significant proportion in Ibadan.

Retinal vascular occlusion is another significant finding of our study. Studies showed a strong relationship between systemic hypertension and retinal vascular occlusion. The Eye Disease Case-Control Study Group found that having a medical history including hypertension increased the odds of having CRVO by $110 \% .{ }^{24}$ Hypertensive patients have atherosclerosis and arteriovenous crossing abnormalities predisposing to venous obstruction. In CRVO, the artery and vein share the same adventitia in the optic nerve where the arterosclerotic artery compresses the vein ${ }^{25}$; this predisposes to thrombus formation in the vein. ${ }^{26}$ Branch retinal vein occlusion results at arteriovenous crossings. Systemic hypertension is a risk factor. ${ }^{27}$ Hemicentral retinal vein occlusion is an emerging presentation noted in this study. Congenital anomaly of the central retinal vein occurs, whereby the two quadrants from the superior retina and inferior retina each drain into separate veins before eventually entering the central retinal vein. Obstruction in either vein produces a hemicentral stasis seen as inferior or superior half widespread hemorrhages. $^{28}$

Retinal artery occlusion is a devastating condition producing profound visual loss. In some of our patients, a cilioretinal artery supplying the macular area was spared in the obstruction. Systemic hypertension is a risk factor for retinal artery occlusion. ${ }^{29}$ 

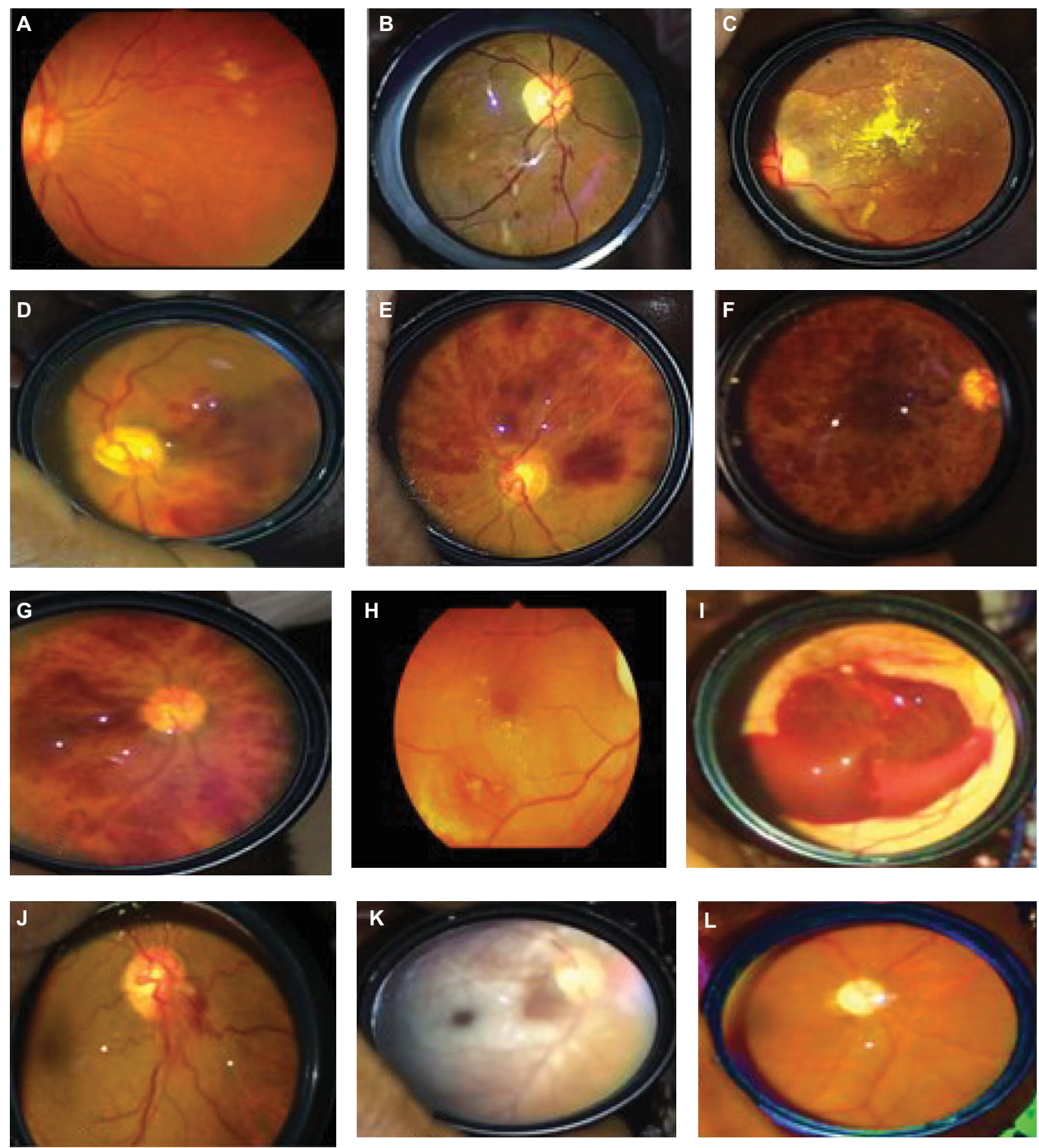

Figure I Retinal vascular changes in hypertensives in lbadan

Notes: (A and B) Hypertensive retinopathy, (C) hypertensive choroidopathy with macular exudates, (D) branch retinal vein occlusion, (E) superior hemicentral retinal vein occlusion, (F) ischemic CRVO, (G) nonischemic CRVO, (H) leaking RAM, (I) ruptured RAM, (J) nonarteritic optic neuropathy, (K) central retinal artery occlusion with sparing of cilioretinal artery, and (L) hypertensive optic atrophy.

Abbreviations: CRVO, central retinal vein occlusion; RAM, retinal artery macroaneurysm.

Optic atrophy from HT optic neuropathy and ischemic optic neuropathy is found in our study. HT optic neuropathy is a recognized entity. Vasospasm and loss of autoregulation in anterior ciliary vessels occur in systemic hypertension. ${ }^{30}$ Secondary optic atrophy is the end result.

RAM occurs in uncontrolled hypertensives..$^{31}$ Increased blood pressure causes damage to the retinal arteriolar vessel wall, thereby producing fusiform dilatation and eventual rupture. Most patients with RAM present with retinal and vitreous hemorrhage and retinal exudation that may extend to the macula with a resultant visual loss. Hypertensive choroidopathy occurs in severe hypertension and is characterized by macular star and exudative retinal detachment involving the posterior pole. ${ }^{32}$

Hemorrhagic choroidal detachment was seen in two patients with severe hypertension. The condition is associated with severe hypertension. ${ }^{33}$ The prognosis is very poor despite prompt surgical intervention to drain the blood.

There is a positive correlation between LV RWT and severity of retinopathy in both eyes in this study. In a nested case-control analysis of the Beaver Dam Eye Study, individuals with hypertensive retinopathy (retinal microaneurysms and retinal hemorrhages) were twice as likely to die from cardiovascular events as those without these signs. ${ }^{34}$ Earlier 
study from Asia showed no significant relationship between LVH severity and retinopathy ${ }^{35}$; however, the presence of retinopathy in a hypertensive patient should alert the clinician about the risks of a fatal outcome.

\section{Conclusion}

Hypertensive retinopathy and retinal vascular changes are common in hypertensive patients in Ibadan. Regular eye examination is advocated. There should be greater communication between ophthalmologists and primary care physicians. This interdisciplinary cooperation will aid in identifying patients most at risk for visual loss and death, and therefore urgent candidates for the existing treatments.

\section{Acknowledgments}

We wish to express our gratitude to our sponsors the Osuntokun Research Grant and the University of Ibadan Senate Research Grant for the support given for this study. We thank our patients for consenting to participate in this study. We also appreciate the resident doctors and nursing staff who helped the patients. Victor Ilevioda, the research assistant, is also appreciated for the support.

\section{Disclosure}

The authors report no conflicts of interest in this work.

\section{References}

1. Ogah OS. Hypertension in Sub Sahara African populations: the burden of hypertension in Nigeria. Ethn Dis. 2006;16(4):765.

2. Opadijo OG, Omotoso ABO, Akande AA. Relation of electrocardiographic left ventricular hypertrophy to blood pressure, body mass index, serum lipids and blood sugar levels in adult Nigerians. Afr J Med Med Sci. 2003;32(4):395-399.

3. Ike SO, Onwubere BJ. The relationship between diastolic dysfunction and level of blood pressure in Blacks. Ethn Dis. 2003;13(4):463-469.

4. Falase AO, Ayeni O, Sekoni GA, Odia OJ. Heart failure in Nigerian hypertensives. Afr J Med Sci. 1983;12(1):7-15.

5. Falase AO, Cole TO, Osuntokun BO. Myocardial infarction in Nigerians. Trop Geogr Med. 1974;25(2):147-150.

6. Osuntokun BO, Bademosi O, Akinkugbe OO, Oyediran AB, Carlisle R. Incidence of stroke in an African city: results from the stroke registry at Ibadan, Nigeria, 1973-1975. Stroke. 1979;10(2):205-207.

7. Akinkugbe OO. Tropical nephropathy - an overview. Afr J Med Med Sci. 1992;21(1):3-7.

8. Wong TY, McIntosh R. Hypertensive retinopathy signs as risk indicators of cardiovascular morbidity and mortality. Br Med Bull. 2005;73-74(1):57-70.

9. Wang $\mathrm{S}, \mathrm{Xu} \mathrm{L}$, Jonas JB, et al. Retinal vascular abnormalities in adult Chinese in rural and urban Beijing: the Beijing Eye Study. Ophthalmology. 2006;113(10):1752-1757.

10. Rodríguez NA, Zurutuza A. Ophthalmological manifestations of arterial hypertension. An Sist Sanit Navar. 2008;31(suppl 3):13-22.

11. Cuspidi C, Negri F, Giudici V, Sala C. Retinal changes and cardiac remodelling in systemic hypertension. Ther Adv Cardiovasc Dis. 2009;3(3):205-214.
12. Sagar S, Rosangluaia S, Kumari S, Sharma BK. Proteinuria in essential hypertension. Indian J Med Res. 1991;94:50-54.

13. Baker ML, Hand PJ, Wang JJ, Wong TY. Retinal signs and stroke: revisiting the link between the eye and brain. Stroke. 2008;39(4): 1371-1379.

14. Cuspidi C, Meani S, Salerno M, et al. Retinal microvascular changes and target organ damage in untreated essential hypertensives. J Hypertens. 2004;22(11):2095-2102.

15. Cuspidi C, Macca G, Salerno M, et al. Evaluation of target organ damage in arterial hypertension: which role for qualitative funduscopic examination? Ital Heart J. 2001;2(9):702-706.

16. Shantha GP, Srinivasan Y, Kumar AA, et al. Can retinal changes predict coronary artery disease in elderly hypertensive patients presenting with angina? Am J Emerg Med. 2010;28(5):617-621.

17. Sharp PS, Chaturvedi N, Wormald R, McKeigue PM, Marmot MG, Young SM. Hypertensive retinopathy in Afro- Caribbeans and Europeans: prevalence and risk factor relationships. Hypertension. 1995;25(6): $1322-1325$.

18. Akinkugbe OO. The rarity of hypertensive retinopathy in the African. Am J Med. 1968;45(3):401-404.

19. Ladipo GO. Hypertensive retinopathy in Nigerians. A prospective clinical study of 350 cases. Trop Geogr Med. 1981;33(4):311-316.

20. Lwanga SK, Lemeshow S. Sample Size Determination in Health Studies: A Practical Manual. Geneva: World Health Organization; 1991.

21. Lang RM, Badano LP, Mor-Avi V, et al. Recommendations for chamber quantification. Eur J Echocardiogr. 2006;7(2):79-108.

22. Busari OA, Opadijo OG, Omotoso AB. Microalbuminuria and hypertensive retinopathy among newly diagnosed nondiabetic hypertensive adult Nigerians. Niger J Clin Pract. 2011;14(4):436-439.

23. Omolase CO, Adekanle O, Omolase BO. Hypertensive retinopathy in a Nigerian Community. Sudan Med J. 2010;46(2):79-84.

24. The Eye Disease Case-Control Study Group. Risk factors for central retinal vein occlusion. Arch Ophthalmol. 1996;114(5):545-554.

25. Scheerer R. UeberVeranderungen der ZentralvenebeiGlaukomatosenZustanden des Scnervenkofes und uberKollateralbildungimBereich des Vorderen des Zentralnervenstammes. Arch F Ophth. 1992;110:293.

26. Green WR, Chan CC, Hutchins GM, Terry JM. Central retinal vein occlusion: a prospective histopathologic study of 29 eyes in 28 cases. Retina. 1981;1(1):27-55.

27. Johnston RL, Brucker AJ, Steinmann W, Hoffman ME, Holmes JH. Risk factors of branch retinal vein occlusion. Arch Ophthalmol. 1985;103:1831-1832.

28. Hayreh SS. Hemi central retinal vein occlusion: pathogenesis, clinical features, natural history. Arch Ophthalmol. 1980;98(9):1600-1609.

29. Destro M, Gragoudas E. Arterial occlusions. In: Albert DM, Jakobiec FA, editors. Principles and Practice of Ophthalmology. Vol. 3. Philadelphia: W.B. Saunders Company; 2000:1879-1887.

30. Hayreh SS, Servais GE, Virdi PS. Fundus lesions in malignant hypertension: V. Hypertensive optic neuropathy. Ophthalmology. 1986;93(1):74-87.

31. Lewis RA, Norton EWD, Gass JDM. Acquired arterial macroaneurysms of the retina. Br J Ophthalmol. 1976;60:21-30.

32. Hayreh SS, Servais GE, Virdi PS. Fundus lesions in malignant hypertension: V. Hypertensive choroidopathy. Ophthalmology. 1986;93(1):1383-1400.

33. Yang SS, Fu AD, McDonald HR, Johnson RN, Ai E, Jumper JM. Massive spontaneous choroidal hemorrhage. Retina. 2003;23(2):139-144.

34. Wong TY, Klein R, Nieto FJ, et al. Retinal microvascular abnormalities and ten-year cardiovascular mortality. A population-based case-control study. Ophthalmology. 2003;110(5):933-940.

35. Shirafkan A, Motahari M, Mojerlou M, Rezghi Z, Behnampour N, Gholamrezanezhad A. Association between left ventricular hypertrophy with retinopathy and renal dysfunction in patients with essential hypertension. Singapore Med J. 2009;50(12):1177-1183. 


\section{Publish your work in this journal}

The International Journal of General Medicine is an international, peer-reviewed open-access journal that focuses on general and internal medicine, pathogenesis, epidemiology, diagnosis, monitoring and treatment protocols. The journal is characterized by the rapid reporting of reviews, original research and clinical studies across all disease areas.
The manuscript management system is completely online and includes a very quick and fair peer-review system, which is all easy to use. Visit http://www.dovepress.com/testimonials.php to read real quotes from published authors.

Submit your manuscript here: https://www.dovepress.com/international-journal-of-general-medicine-journal 